\title{
Global energy sector emission reductions and bioenergy use: overview of the bioenergy demand phase of the EMF-33 model comparison
}

\author{
Nico Bauer ${ }^{1}$ - Steven K. Rose $^{2}$ • Shinichiro Fujimori ${ }^{3,4}$. \\ Detlef P. van Vuuren ${ }^{5,6} \cdot$ John Weyant $^{7} \cdot$ Marshall Wise $^{8} \cdot$ Yiyun Cui $^{8}$. \\ Vassilis Daioglou $^{5}$ • Matthew J. Gidden ${ }^{9}$ - Etsushi Kato ${ }^{9}$ • \\ Alban Kitous $^{11}$ - Florian Leblanc ${ }^{12}$ - Ronald Sands ${ }^{13}$ - Fuminori Sano ${ }^{14}$. \\ Jessica Strefler ${ }^{1}$ - Junichi Tsutsui ${ }^{15}$ - Ruben Bibas ${ }^{12}$ • Oliver Fricko ${ }^{9}$. \\ Tomoko Hasegawa $^{4}$ - David Klein ${ }^{1}$ - Atsushi Kurosawa ${ }^{10}$. \\ Silvana Mima ${ }^{16} \cdot$ Matteo Muratori $^{17}$
}

Received: 1 November 2017 / Accepted: 15 May 2018 / Published online: 2 July 2018

(C) Springer Science+Business Media B.V., part of Springer Nature 2018

\begin{abstract}
We present an overview of results from 11 integrated assessment models (IAMs) that participated in the $33^{\text {rd }}$ study of the Stanford Energy Modeling Forum (EMF-33) on the viability of large-scale deployment of bioenergy for achieving long-run climate goals. The study explores future bioenergy use across models under harmonized scenarios for future
\end{abstract}

This article is part of the special issue "Assessing Large-scale Global Bioenergy Deployment for Managing Climate Change (EMF-33)" edited by Steven Rose, John Weyant, Nico Bauer, Shinichiro Fuminori, Petr Havlik, Alexander Popp, Detlef van Vuuren, and Marshall Wise.

Electronic supplementary material The online version of this article (https://doi.org/10.1007/s10584-0182226-y) contains supplementary material, which is available to authorized users.

Nico Bauer

nico.bauer@pik-potsdam.de

1 Potsdam Institute for Climate Impact Research (PIK), Leibniz Association, 601203, 14412,

Potsdam, Germany

2 Energy and Environmental Analysis Research Group, Electric Power Research Institute, Washington, DC, USA

3 Department of Environmental Engineering, Kyoto University, Kyoto, Japan

4 National Institute for Environmental Studies (NIES), Tsukuba, Japan

5 Netherlands Environmental Assessment Agency (PBL), The Hague, The Netherlands

6 Copernicus Institute for Sustainable Development, Utrecht University, Utrecht, The Netherlands

7 Stanford University, Palo Alto, CA, USA

$8 \quad$ Pacific Northwest National Laboratory (PNNL), College Park, MD, USA 
climate policies, availability of bioenergy technologies, and constraints on biomass supply. This paper provides a more transparent description of IAMs that span a broad range of assumptions regarding model structures, energy sectors, and bioenergy conversion chains. Without emission constraints, we find vastly different $\mathrm{CO}_{2}$ emission and bioenergy deployment patterns across models due to differences in competition with fossil fuels, the possibility to produce large-scale bio-liquids, and the flexibility of energy systems. Imposing increasingly stringent carbon budgets mostly increases bioenergy use. A diverse set of available bioenergy technology portfolios provides flexibility to allocate bioenergy to supply different final energy as well as remove carbon dioxide from the atmosphere by combining bioenergy with carbon capture and sequestration (BECCS). Sector and regional bioenergy allocation varies dramatically across models mainly due to bioenergy technology availability and costs, final energy patterns, and availability of alternative decarbonization options. Although much bioenergy is used in combination with CCS, BECCS is not necessarily the driver of bioenergy use. We find that the flexibility to use biomass feedstocks in different energy sub-sectors makes large-scale bioenergy deployment a robust strategy in mitigation scenarios that is surprisingly insensitive with respect to reduced technology availability. However, the achievability of stringent carbon budgets and associated carbon prices is sensitive. Constraints on biomass feedstock supply increase the carbon price less significantly than excluding BECCS because carbon removals are still realized and valued. Incremental sensitivity tests find that delayed readiness of bioenergy technologies until 2050 is more important than potentially higher investment costs.

\section{Introduction}

The future of bioenergy use recently became a highly controversial topic. Several studies identified bioenergy as a substitute for fossil fuels and a potentially crucial option to reduce greenhouse gas (GHG) emissions from the energy sector for long-run climate objectives. Moreover, bioenergy combined with carbon capture and storage (i.e., BECCS) allows for carbon dioxide removals (CDR) to offset $\mathrm{CO}_{2}$ emissions and potentially reverse warming by means of net negative emissions. The interactions of bioenergy deployment and emission reductions were part of previous model comparison exercises and single-model studies (Grahn et al. 2007; Rose et al. 2014; van Vuuren et al. 2010) and assessment reports (Chum et al. 2011; Clarke et al. 2014). Despite the large variations in total bioenergy use

\footnotetext{
9 International Institute for Applied Systems Analysis (IIASA), Laxenburg, Austria

10 The Institute of Applied Energy, Minato, Tokyo 105-0003, Japan

11 Joint Research Center (JRC), Seville, Spain

12 Centre International de Recherche sur l'Environnement et le Développement, Paris, France

13 U.S. Department of Agriculture, Economic Research Service, Washington, DC, USA

14 Research Institute of Innovative Technology for the Earth (RITE), Kyoto, Japan

15 Environmental Science Laboratory, Central Research Institute of Electric Power Industry (CRIEPI), Abiko, Chiba, Japan

16 Grenoble Applied Economics Lab (GAEL), University Grenoble Alpes, CNRS, INRA, Grenoble INP, Grenoble, France

17 National Renewable Energy Laboratory (NREL), Golden, CO, USA
} 
and deployment patterns, various studies found that bioenergy is valuable in scenarios achieving ambitious climate change targets, and highlighted the potential role of BECCS (Klein et al. 2014; Kriegler et al. 2014; Rose et al. 2014).

This raised critique about assumptions on, among others, biomass feedstock supply and bioenergy technologies availability in integrated assessment models (Anderson and Peters 2016; Buck 2016; Field and Mach 2017; Fuss et al. 2014). Moreover, large-scale bioenergy production can lead and intensify various issues such as direct and indirect land-use GHG emissions, competition with food production, threats to biodiversity, and social conflicts on many issues such as land tenureship (Creutzig et al. 2013; Fargione et al. 2010). The main focus of this study is biomass use whereas biomass production systems are treated in a companion paper (Rose et al., 2018, Global biomass supply modeling for long-run management of the climate system, this Issue, in preparation).

This paper represents an overview of results from 11 integrated assessment models (IAMs) that participated in the 33rd study of the Stanford Energy Modeling Forum (EMF-33). Building on EMF-27 (Kriegler et al. 2014; Rose et al. 2014), this study focuses on the three-sided relationship between $\mathrm{CO}_{2}$ emission limitations, bioenergy use, and advanced bioenergy technologies (ABTs). ABTs include options to convert ligno-cellulosic biomass into modern energy carriers such as electricity, liquids, and hydrogen (incl BECCS variants). The EMF-33 study brings together IAMs that represent a range of modeling approaches and that explicitly consider alternative biomass feedstock uses in the energy sector and at least one BECCS option.

The EMF-33 scenario protocol explores a set of assumptions along two dimensions. First, the emission reduction requirements vary in strength. Specifically, the cumulative 2011-2100 emission limitations include scenarios that help to inform the well-below 2 and $1.5{ }^{\circ} \mathrm{C}$ objectives mentioned in the Paris Agreement (see also Rogelj et al. 2015). The second dimension considers biomass feedstock supply and ABT availability. Limitations on annual biomass feedstock availability are implemented by imposing an upper limit. ABTs are not yet fully mature for commercial deployment and large-scale ramp-up, although a few small-scale projects are in operation (Bhave et al. 2017; Lomax et al. 2015). Variations of future ABT availability are explored by (i) excluding certain technology options such as BECCS, (ii) delaying technology availability, or (iii) increasing technology costs. These variations require changes to the scale and structure of the energy sector in general and bioenergy use in particular to comply with the carbon budget. EMF-27 tested the availability of all CCS technologies (incl fossil CCS). EMF-33 goes a step further and differentiates exclusion of specific ABTs and tests the more gradual sensitivity of ABT maturity.

This EMF-33 overview on bioenergy deployment presents key results and contributes to two major insights. First, we improve the transparency of models and scenarios and relate differences in results to differences in model assumptions focusing on bioenergy use. Second, we revise previous bioenergy deployment quantifications as well as provide new quantifications and analyze the role of bioenergy and ABTs in the context of global emission limitations. The study's large set of models allows us to explore the uncertainty space of future bioenergy deployment patterns and identify crucial assumptions that affect results to improve transparency, understanding, and evaluation of models and scenarios. This overview is complemented by thematic EMF33 studies on techno-economic specifications of ABTs (Daioglou et al. 2018, Bioenergy technologies and climate change mitigation pathways, this Issue, in preparation), BECCS (Muratori et al., 2018, Bioenergy and carbon capture and storage (BECCS): results from the EMF-33 study, this Issue, in preparation), bioenergy and transportation (Bibas et al., 2018, Bioenergy contribution to the transport sector decarbonization: a multi-model assessment, this Issue, in preparation.), and international trade in bioenergy (Daioglou et al. 2018, Implications of climate change mitigation strategies on international bioenergy trade, this Issue, submitted). 


\section{Methods}

\subsection{EMF-33 models}

Eleven IAMs contribute to EMF-33. All participated in model comparisons (e.g., Bauer et al. 2017; Riahi et al. 2017; Kriegler et al. 2014; Rose et al. 2014).

Table 1 presents key model characteristics that are most relevant to understand scenarios of bioenergy use. The models represent in detail the development and restructuring of the energy sector that is influenced by policies. The models take care of system-relevant boundaries and limitations such as geological storage and land availability. ABTs are considered in all models with varying breadth and detail. All models comprise bio-electricity with CCS based on integrated gasification, but not all models assume that bioenergy-to-liquids with CCS is available. A subset of models represents hydrogen production fueled with biomass, also using ligno-cellulosic feedstocks, both with and without a CCS option. Other conversion routes in Table 1 are not considered ABTs in this study.

Secondary energy carriers (i.e., fuels) derived from bioenergy are combined with fuels derived from other primary energy sources to supply final energy. The flexibility to swap fuels is an important modeling assumption for bioenergy demand development (Kaya et al. 2017). Models with non-linear sharing (e.g., logit, CES...) derive scenarios with stable energy mixes inherited by the historic status quo. Price changes lead to smooth changes of energy mixes. Models with full flexibility shift fuel mixes towards the cheapest cost alternative subject to constraints on ramping-up of new capacities or explicit integration limits. Hence, these models derive scenarios with endogenous successions of competitive technologies.

All models but two (IMAGE, POLES) represent land-use competition between biomass production and crop, forest, and pasture land. IMAGE allows bioenergy expansion only in non-commercial land (such rest lands).

\subsection{The scenario protocol}

The scenario protocol is summarized in Table S1, along with a summary of the scenarios submitted for each model. The scenario protocol harmonized definitions and variations of carbon budgets and availability of bioenergy technologies. Moreover, definitions for data reporting are harmonized.

Due to the study's energy sector focus, we harmonized budgets on cumulative $\mathrm{CO}_{2}$ emissions 2011-2100 from sources from the energy and industry sectors net of CDR by BECCS. Temporary over-shooting of the budget is allowed. Different to Rogelj et al. (2015), $\mathrm{CO}_{2}$ emissions from the land-use sector and non- $\mathrm{CO}_{2} \mathrm{GHG}$ emissions are not accounted for in the budget. Afforestation is included as an additional CDR option in some models, but it does not offset $\mathrm{CO}_{2}$ emissions of the energy and industry budget. To manage intersectoral carbon leakage, we apply the carbon price resulting from the energy sector budget constraint to consistently value land-use $\mathrm{CO}_{2}$ and all other GHG emissions using $\mathrm{CO}_{2}$-equivalent emission taxes. This can affect biomass supply (i) penalizing direct and indirect land-use sector emissions and (ii) incentivizing afforestation that competes with biomass for land.

The intertemporal carbon budgets are combined with near-term policies until 2020, including Cancun pledges or 2020 objectives in nationally determined contributions (NDCs) as agreed in the Paris Agreement. After 2020, each model implements the policy necessary to achieve a carbon budget (see Table 1). The choice of the carbon budgets is motivated by the 


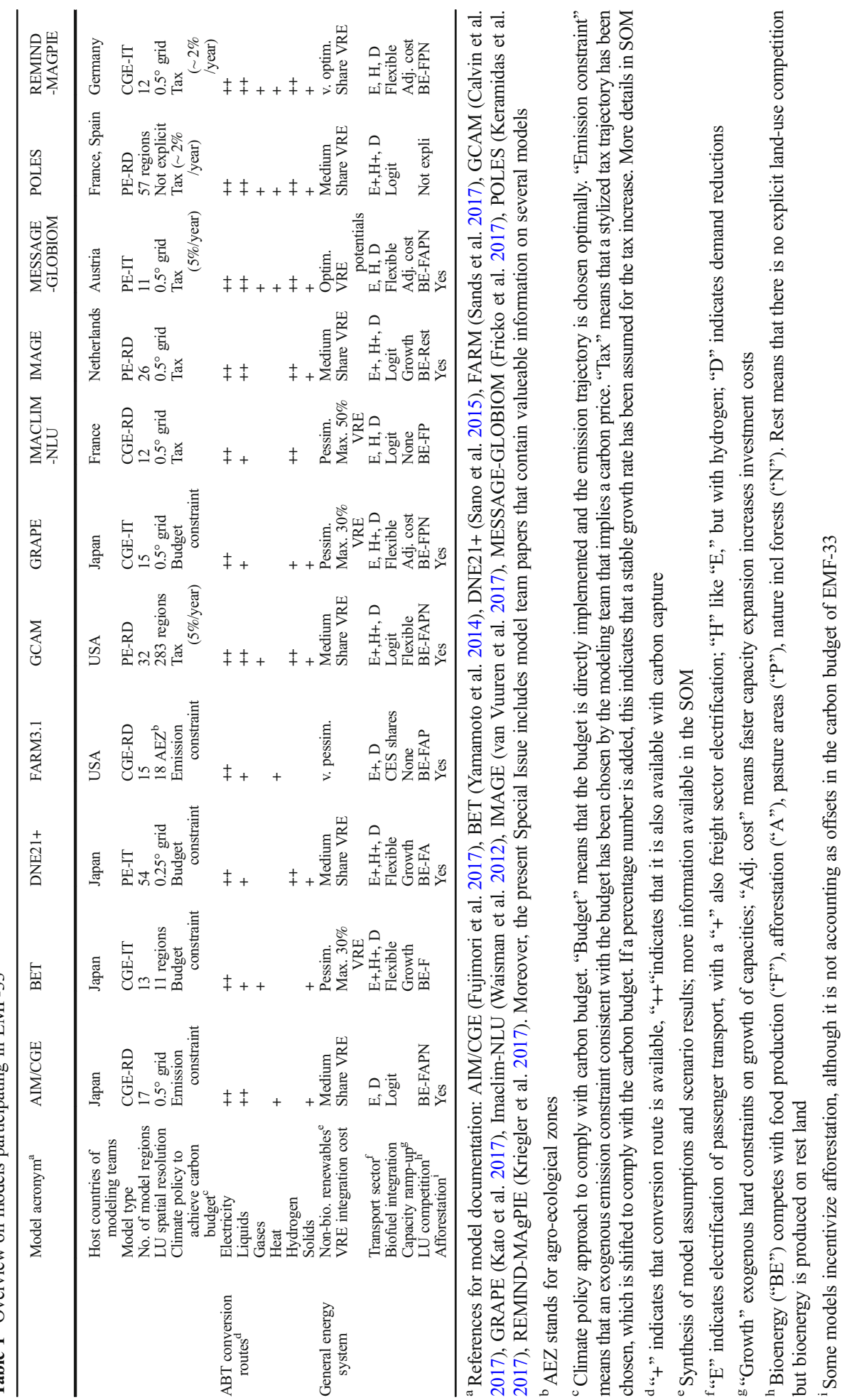


goals of the Paris Agreement (see also Rogelj et al. 2015). The low budget of $1000 \mathrm{GtCO}_{2}$ lies in the range of emissions likely to achieve a $2{ }^{\circ} \mathrm{C}$ target, whereas the very low budget $\left(400 \mathrm{GtCO}_{2}\right)$ aims at the $1.5^{\circ} \mathrm{C}$ target. The high budget $\left(1,600 \mathrm{GtCO}_{2}\right)$, however, is unlikely to achieve the $2{ }^{\circ} \mathrm{C}$ target. Note that total GHG emissions are not harmonized and, thus, climate outcomes vary across models.

The budget scenarios are first run with full availability of ABTs and default assumptions of biomass feedstock availability. Both assumptions are varied along with the stringency of the budgets. The variations of ABTs consider the exclusion of (i) all ABTs, of (ii) BECCS technologies, and of (iii) ABTs that convert ligno-cellulosic feedstocks into liquid fuels. These three exclusion scenarios are complemented by two more gradual variations. First, we increased the investment costs of all ABTs by $100 \%$ and, secondly, we delayed the readiness of ABT deployment to 2050. Finally, we limited global biomass feedstock supply for modern uses to $100 \mathrm{EJ} /$ year.

Over 165 baseline and $\mathrm{CO}_{2}$ budget scenarios were submitted for EMF-33, but the number of submissions varying by model, with a low of five scenario submissions and a high of 20 (Table S1). All models ran the high and low budget scenarios with their full set of default technologies, bioenergy, and non-bioenergy, and ten ran the very low budget scenario, but not all were able to find a feasible solution. An infeasible (unachievable) solution means that, given the models assumptions about economic growth, techno-economics, biomass supply constraints, technology deployment dynamics, and the models solution algorithm, the model could not produce a viable emissions path that satisfied the emissions budget constraint. Limitations on bioenergy technology and feedstock supply reduced the number of models finding feasible solutions for both the low and the very low budgets.

\section{Results}

\section{1 $\mathrm{CO}_{2}$ emissions and bioenergy use}

The main socioeconomic drivers of future energy demand (see Figure S3) are relatively similar across models and are similar to SSP2-like assumptions (Riahi et al. 2017). Baseline $\mathrm{CO}_{2}$ emissions from energy and industry (Fig. 1a) vary significantly across models and show diverse patterns resulting in cumulative 2011-2100 $\mathrm{CO}_{2}$ emissions ranging from 3600 to $5,500 \mathrm{GtCO}_{2}$. Some models feature slight growth and stabilize around $50 \mathrm{GtCO}_{2} /$ year whereas other models project continued growth exceeding $80 \mathrm{GtCO}_{2} /$ year in 2100 (e.g., MESSAGEGLOBIOM).

Generally, high baseline $\mathrm{CO}_{2}$ emissions represent abundant fossil fuels and thus bioenergy use is limited (Fig. 1b). Conversely, scarce fossil fuel availability depresses growth of final energy use, implying greater demand for non-fossil energy sources like bioenergy that can exceed $100 \mathrm{EJ} /$ year by 2050 and reach nearly $200 \mathrm{EJ} / \mathrm{year}$ by 2100 . Consequently, higher final energy use across models in 2100 is not necessarily associated with higher bioenergy use (see Figure S5). Bioenergy is not necessarily the only non-fossil alternative; non-bioenergy renewables and nuclear can serve as alternatives in the energy sector (see Figure S14).

Implementing a carbon budget strongly limits the variation in future emission pathways, but increases the range of bioenergy use across models (Fig. 1c). But still, emission profiles vary across models with more than $20 \mathrm{GtCO}_{2}$ /year differences in 2100 in the low budget case (Fig. 1b). Some models deploy as much as $280 \mathrm{EJ} /$ year of bioenergy by 2050 . However, 
for the high-budget case, only two models exceed $150 \mathrm{EJ} / \mathrm{year}$. For the low budget, four models go significantly beyond that line and for the very low budget, only one out of six models falls below the 150 EJ/year. For comparison, Chum et al. (2011) identified 100$300 \mathrm{EJ} /$ year in 2050 as a plausible range with the upper end requiring policy efforts regarding efficiency improvements in the agricultural sector and good governance.

Bioenergy use generally grows until 2100 but at slower rates. Some models reach $\sim 400 \mathrm{EJ} / \mathrm{year}$ of bioenergy use. In general, for varying carbon budgets, each model approaches a relatively similar long-term deployment level. Consequently, most models show larger differences in deployment levels across the carbon budgets in 2050 than those in 2100. Few models (GCAM, BET) show stronger long-term than mid-term sensitivity because other $\mathrm{CO}_{2}$ emission mitigation options are more competitive around 2050. Across models, we find that more ambitious mid-term emission limitations lead to higher bioenergy use in the mid-term, whereas delayed emission mitigation and higher removals towards the end of the century increase bioenergy use in 2100 (see Figure S6). In summary, stronger emission limitations generally lead to higher bioenergy use within and across models and, thus, the development of $\mathrm{CO}_{2}$ emissions and bioenergy use are interrelated.

Models differ in biomass supply assumptions. High bioenergy use is associated with models with low afforestation activity and eventually high bioenergy crop yield rates (REMIND-MAgPIE, GRAPE). Large afforestation can put a limit on available land for biomass feedstock growth (AIM/CGE). Only the GCAM model combines high bioenergy deployment with high afforestation (see SOM for details). Models like POLES, MESSAGE-GLOBIOM, and IMAGE impose constraints on land availability that effectively limit biomass potential. This leads to increasing biomass feedstock prices as growing demands face limited supply (see Table S4 and the EMF-33 biomass supply assessment for more insights and information on biomass supply modeling (Rose et al., 2018, Global biomass supply modeling for long-run management of the climate system, this Issue, in preparation).

The challenge to integrate biofuels into the secondary energy mix depends on the assumed system flexibility. If biofuel integration is challenging, which is represented by non-linear sharing approaches, total bioenergy use is sensitive to the energy market size and, thus, to reductions of fossil fuel consumption. As fossil fuel use needs to decrease and bioenergy is a complement to rather than a substitute for fossil fuels, absolute bioenergy use declines while its relative share increases. The market size effect is stronger with less flexible substitution (Imaclim-NLU) and tighter carbon budgets (AIM/CGE, POLES).

The regional allocation of modern bioenergy use includes significant trade flows (Figures S11 and S12). Economically affluent OECD countries show the highest bioenergy use per capita in both baseline and carbon budget scenarios. Therefore, OECD countries use a significant share of global bioenergy. Asia shows much smaller per-capita modern bioenergy use, but its large population leads to large total use. In most models, Latin America exports biomass feedstocks to OECD and Asia. Despite large land areas, Reforming Economies in Eastern Europe and the Former Soviet Union are no significant exporter in most models (see Daioglou et al. 2018, Implications of climate change mitigation strategies on international bioenergy trade, this Issue, submitted; Rose et al., 2018, Global biomass supply modeling for long-run management of the climate system, this Issue, in preparation).

\subsection{Specialization patterns of bioenergy use}

The models allocate biomass to different conversion routes suggesting different ABT deployment and broader energy sector development (see Figures S14-S18). Biomass feedstocks are allocated to deliver the highest value, which depends on various assumptions: availability of bioenergy and 
non-bioenergy technology alternatives, their techno-economic parameters, prices and final energy demands, constraints on investment dynamics, and the strength of climate policies.

The question of bioenergy allocation is mostly a compromise between electricity and liquid fuel production (Fig. 2). Biomass electricity production features high carbon capture rates and low energy conversion efficiency, and non-bioenergy decarbonization opportunities are relatively abundant in many models (e.g., renewables, nuclear). Liquid fuel production, however, captures a smaller fraction of the carbon content of biomass but the energy conversion efficiency is relatively high and liquid fuel production is difficult to decarbonize while transport energy and service demands grow.

In baseline scenarios, no model allocates significant amounts of bioenergy to electricity generation, whereas bio-liquid production is more competitive (up to $45 \mathrm{EJ} / \mathrm{year}$ in 2050). In the longer run, this discrepancy increases, because fossil fuels (particularly oil) get scarcer in most models and bio-liquid production can substitute fossils, e.g., AIM/CGE, GRAPE, Imaclim-NLU, and POLES (see Table S3). Models applying non-linear sharing approaches for modeling energy mixes (AIM/ CGE, GCAM, IMAGE, POLES) allocate bioenergy more evenly between liquid fuel and electricity.

Carbon budgets boost BECCS deployment, but sectoral allocations vary significantly. If BECCS is only available for electricity production, it expands strongly (BET, DNE21+, GRAPE). The bio-electricity share in the electricity mix remains limited (see Figure S16). GRAPE shows by far the highest share, partly due to a hard limit on the share of nonbioenergy renewables, but remains below 50\%. High deployment of BECCS in the electricity sector is associated to relatively high oil consumption (see Figure S14), because the high capture rate offsets the $\mathrm{CO}_{2}$ emissions. Only the models FARM and Imaclim-NLU show reluctance to expand the electricity BECCS option.

If also bio-liquid with CCS is available, three models specialize into it (GCAM, MESSAGE-GLOBIOM, and REMIND-MAgPIE). These models are equipped with large potentials for low-carbon electricity generation making bio-liquid production the more attractive alternative (see Figure S16). AIM/CGE, IMAGE, and POLES show a relatively balanced mix of bio-electricity and bio-liquid. IMAGE and POLES assume more challenges in renewable electricity integration, and therefore bio-electricity is deployed. Although significant amounts of biomass feedstocks are used for electricity generation in this subset of models, its share in the total electricity mix remains small $(<10 \%)$. Despite huge biomass input for liquids production, in all models, transportation sector decarbonization also relies on developing new drive trains based on electricity, hydrogen, and gases (see Figure S18).

Some models project significant electricity sector decarbonization under baseline conditions and, thus, EMF-33 models project dramatically different sectoral baseline $\mathrm{CO}_{2}$ emissions. We find that a larger sectoral share of baseline emissions budget is correlated with a greater share of bioenergy deployment for models with similar bioenergy options available (Figure S8). For instance, a larger electricity share of baseline $\mathrm{CO}_{2}$ emissions corresponds with deploying a larger fraction of bioenergy in electricity (e.g., GCAM, IMAGE); the inverse also holds (e.g., REMIND-MAGPIE).

\subsection{CCS and bioenergy}

The EMF-33 models are ambiguous about the question whether CDR via BECCS deployment triggers additional bioenergy use (Figure S7(a)). Several models expand bioenergy beyond baseline levels to combine it with CCS (e.g., GRAPE, REMIND-MAgPIE). Other models partly reallocate baseline bioenergy use to combine it with CCS, for instance, in 2050 allowing CDR expansion up to $5 \mathrm{GtCO}_{2} /$ year, but bioenergy use increases by less than $50 \mathrm{EJ} /$ year. In some models (e.g., POLES), 

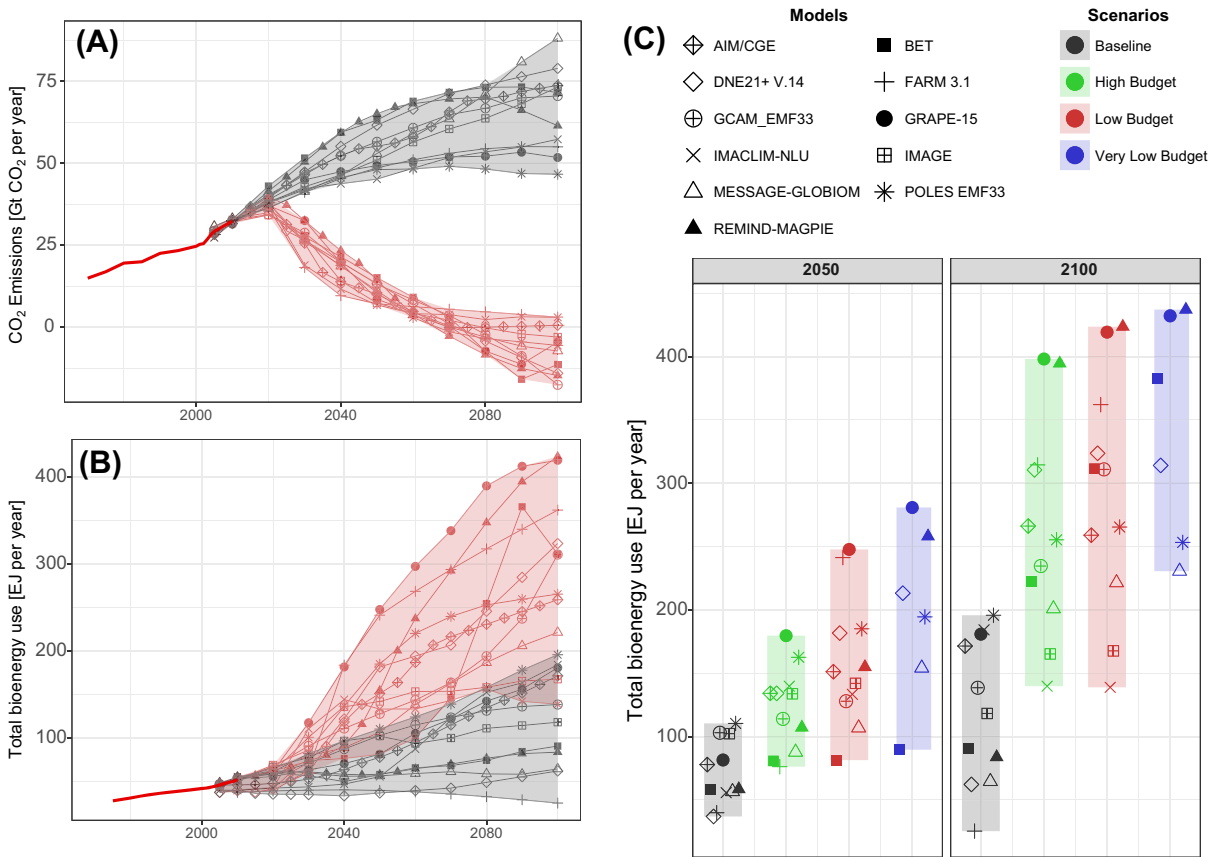

Fig. 1 Overview on global $\mathrm{CO}_{2}$ emissions from energy and industry (a) and total global bioenergy deployment $(\mathbf{b}, \mathbf{c})$ in scenarios with full technology availability. Includes traditional bioenergy use

CDR deployment becomes increasingly constrained as carbon budgets get more stringent. Thus, additional bioenergy primarily increases low-carbon energy supply but not carbon removal.

The comparison of bioenergy use in baseline and policy cases is relevant for the economics of BECCS. If bio-liquid production is projected to become competitive with fossil fuels, the additional effort to upgrade and operate the CCS equipment is relatively small. As Daioglou et al. (2018, Bioenergy technologies and climate change mitigation pathways, this Issue, in preparation) point out, models assume additional costs (18\%, ranging 10-43\%) and efficiency losses (9\%, ranging 4-14\%) are relatively small (compared to bio-liquid plant without CCS). Therefore, BECCS can start to penetrate the system at relatively small carbon prices below 30 USD per $\mathrm{tCO}_{2}$ as highlighted by Muratori et al. (2018, Bioenergy and carbon capture and storage (BECCS): results from the EMF-33 study, this Issue, in preparation).

Cumulative CDR by BECCS ranges between 600 and $1300 \mathrm{GtCO}_{2}$ if the very low-budget case is imposed (Figure S7(b)). In the low-budget case, 5 out of 11 models remove at maximum $500 \mathrm{GtCO}_{2}$ from the atmosphere (one model exceeds $1000 \mathrm{GtCO}_{2}$ ). With higher cumulative $\mathrm{CDR}$, also the overshoot beyond the carbon budget limit increases. In the very low-budget case, the overshoot ranges between 345 and $615 \mathrm{GtCO}_{2}$ whereas it is no more than $125 \mathrm{GtCO}_{2}$ in the high-budget case. Large CDR deployment does not necessarily imply large overshoots (GRAPE and DNE21+ in Table S5) because CDR also offsets residual emissions. Combined fossil and bioenergy CCS ranges between 700 and $1200 \mathrm{GtCO}_{2}$ in seven models for all carbon budgets. Models with high fossil CCS shares show small baseline fossil fuel prices. In comparison with EMF-27, total CCS decreased (Koelbl et al. 2014) but the share of BECCS increased. EMF-33 scenarios could reach geological storage limits of $1000 \mathrm{GtCO}_{2}$ assessed by Scott et al. (2015), but compared with Dooley (2013), all models are well within the limits of $3900 \mathrm{GtCO}_{2}$. 


\subsection{Sensitivity of ABT and biomass availability}

EMF-27 investigated the sensitivity of total bioenergy use with respect to CCS technology exclusion for both fossil and bioenergy CCS (Rose et al. 2014). EMF-33 revisits this important issue by (i) performing a more focused sensitivity analysis of ABT availability, (ii) explaining the economics behind the results, and (iii) providing a more detailed quantification of different effects. It is important to note upfront that the limitation seriously constrains the emission reduction potentials and, thus, makes many models fail to achieve the low budget and all fail to achieve the very low budget.

The net impact on total bioenergy use in the carbon budget framework is the result of three effects. First, the direct technology effect is simply the reduction of bioenergy use by the ABTs that are excluded. Second, the reallocation effect that shifts biomass supply to the remaining bioenergy technologies. Third, the policy feedback effect that increases $\mathrm{CO}_{2}$ prices required to comply with the carbon budget due to the reduced mitigation portfolio. Higher carbon prices drive bioenergy demand upwards to substitute fossil fuels, unless the marked size effect prevails. However, higher $\mathrm{CO}_{2}$ prices also increase biomass supply costs because direct and indirect land-use emissions of bioenergy are penalized. The demand and supply side policy feedback effects shift bioenergy use to opposite directions with the net effect uncertain.

Figure 3 compares (i) the direct technology (DT) effect and (ii) the combined reallocation and policy feedback (RPF) effect. Generally, the DT is significantly offset in most models by the RPF effect. Particularly, the "No BECCS" scenario indicates that demand for low-carbon energy acts as a major driver for bioenergy use even without the CDR possibility. For instance, models that deploy BECCS at large scale (>100 EJ/year in 2100) reallocate $50 \%$ or more of the reduced bioenergy to alternative conversion routes. In 2050, total bioenergy use can be higher because deeper reductions in fossil fuel use become necessary. The RPF effect can reinforce the DT effect, if the market size effect prevails (negative values in the table for AIM/ CGE and Imaclim-NLU).

The exclusion of advanced bio-liquid production is relevant for a subset of models that show very strong RPF effects that imply only small changes in total bioenergy use. Therefore, models that comprise only a limited portfolio of ABTs without bio-liquid production do not necessarily understate total bioenergy use, but may suggest a bias of its allocation and further biases in the overall energy sector development.

The policy feedback effect on the biomass supply side prevails for REMIND-MAgPIE in 2100. The DT effect in the No BECCS case is approximately the same for both carbon budgets, but the low-budget case shows smaller RPF effect due to much higher biomass prices triggered by higher carbon prices. The SOM provides more information (see Figure S9).

Limiting modern biomass supply to $100 \mathrm{EJ} / \mathrm{year}$ has been considered in EMF-27 already (Rose et al. 2014), but the impacts on BECCS use have not been investigated. The supply side constraint requires more stringent climate policies, which triggers a positive demand side feedback effect on BECCS deployment in 2050 in most models (see Table S6). More bioenergy is allocated to BECCS conversion routes and to alternatives with higher capture rates. For instance, in the high-budget case, most models remove more carbon in 2050 using less biomass. Models that do not increase BECCS deployment (IMAGE and POLES) derive investment decisions in a recursive dynamic model framework and, therefore, do not anticipate the future constraint that triggers upwards adjustment of BECCS investments. 


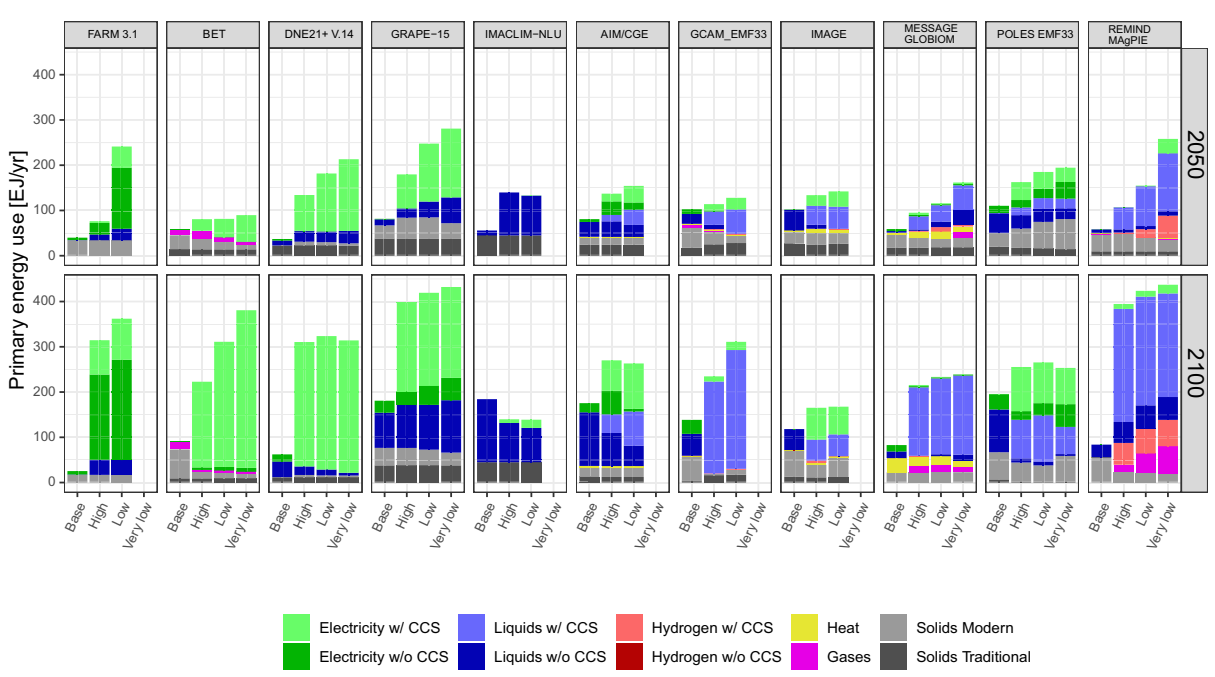

Fig. 2 Allocation of total global primary bioenergy across models and budget scenarios with full technology availability in the years 2050 and 2100 . The five models to the left hand side do not include bio-liquids with $\mathrm{CCS}$, whereas the six models to the right hand side include this technology option

\subsection{Implications on carbon prices}

In the carbon budget framework, varying assumptions on ABTs and biomass supply require changes in $\mathrm{CO}_{2}$ prices to comply with the carbon budget. Models implement carbon prices with very different time profiles that are made comparable by computing the present value (PV) of carbon prices assuming a 5\% annual discount rate (see SOM for methodology and $\mathrm{CO}_{2}$ price paths implemented by the models).

All models show that increasing carbon prices are required for tighter carbon budgets but cover a considerable range (Fig. 4a). For the high- and low-budget cases, most models cluster in a relatively narrow range. At the upper end, the range is pushed by the Imaclim-NLU model that finds decarbonization in the energy sector difficult. At the lower end of the range, the models MESSAGE-GLOBIOM and REMIND-MAgPIE assume plenty of decarbonization alternatives and flexible reconfiguration of the energy sector, although bioenergy use differs considerably. In the low-budget case, six models cluster between 25 and $30 \mathrm{USD} / \mathrm{tCO}_{2}$. These models are also relatively similar in the high-budget case, but in the very low-budget case, three models found the target unachievable and three cover a large range between 80 and $120 \mathrm{USD} / \mathrm{tCO}_{2}$. As models approach the limits of feasible solutions (like the very low budget), carbon prices become highly sensitive.

Figure $4 \mathrm{~b}$ shows for the three ABT exclusion scenarios, the percentage increases of PV carbon prices compared with the case of full technology availability (panel a). In all three sensitivity cases, models find greater impacts on the carbon price as the carbon budgets become more stringent. In case of the high budget, limitations of biomass supply or exclusion of BECCS technologies increases carbon prices by more than $50 \%$ for the majority of models, whereas in the case of the low budget, carbon prices more than double. Models at the upper end of these relative changes use relatively large quantities of bioenergy with BECCS and rely more on CDR like REMIND-MAGPIE, whereas models at the lower end like POLES use less bioenergy with BECCS and rely less on CDR (see Fig. 2). 
The complete exclusion of BECCS or even all ABTs and the significant impacts on carbon prices are useful to isolate some of the potential value of bioenergy, but these are pessimistic assumptions. Therefore, we tested the sensitivity of more moderate changes of the IAMs standard bioenergy assumptions. The exclusion of ABTs for fuel production addresses the sensitivity of a subset of technologies. Also, in standard settings, IAMs generally assume that ABTs are available from 2030 at techno-economic assumptions taken from the literature. We test these assumptions by (i) doubling the investment costs and (ii) delaying the earliest deployment of ABTs to 2050.

The more gradual change in assumptions leads to significantly smaller impacts on carbon prices (panel c). The exclusion of ABTs for fuel production limits increases below $25 \%$ in case of the high and the low budget. The reallocation of bioenergy to alternative uses (see Fig. 3) mutes the impact on carbon prices also for models that allocate biomass feedstock to fuel production. The impact of doubling ABT investment costs is also less than $25 \%$ and reflects the relatively small capital cost share compared with the feedstock costs and the premium generated by CDR realized with BECCS technologies (see Daioglou et al. (2018, Bioenergy technologies and climate change mitigation pathways, this Issue, in preparation)). The impact hardly increases as more stringent budgets are assumed because the relative importance of capital costs decreases. The delayed readiness of ABTs has a small impact on carbon prices for the high carbon budget, but becomes much larger for the low and the very low carbon budget.

\section{Summary and conclusions}

The EMF-33 study quantifies and compares results of 11 global long-term IAMs applying a harmonized scenario protocol focused on advanced bioenergy use and $\mathrm{CO}_{2}$ emission reductions. The model comparison helps to understand scenario results by identifying robust conclusions and relating differences in results to differences in models. The future of global bioenergy use and its role in climate change mitigation scenarios depends on many factors spanning several dimensions of the integrated energy, land-use, and economy system. The most important drivers of bioenergy deployment patterns are assumptions on bioenergy conversion technologies costs and their availability, the potentials and constraints of biomass feedstock supply, and the baseline scenarios that determine total and sectoral emission mitigation requirements. Comparing various IAMs helps to identify robust and general findings as well as crucial assumptions and sticking points that affect the modeling projections. The vast range future bioenergy use and the very significant sensitivities highlight the broad room for maneuvering and the importance of policy making and technology development. Our study also identifies robust findings that refer to key trade-offs that are crucial for policy making.

Without climate policies, the models project $3600-5,500 \mathrm{GtCO}_{2}$ cumulatively emitted 2011-2100. Limiting these emissions to 1600 or 1000 or even $400 \mathrm{GtCO}_{2}$, in line with climate change stabilization ambitions, is challenging. Increasing ABT deployment becomes generally more important for more stringent emission budgets. Particularly, achieving tighter budgets boosts bioenergy deployment by 2050. Also, in the long-run across models, lower $\mathrm{CO}_{2}$ emissions are associated with higher bioenergy use saturating at levels specific to individual models. An inverse sensitivity is observed for some models due to the market size effect that can imply decreasing bioenergy use due to stricter emission limitations, if its integration into the energy system is challenging. Overall, advanced bioenergy use is a robust and valuable 

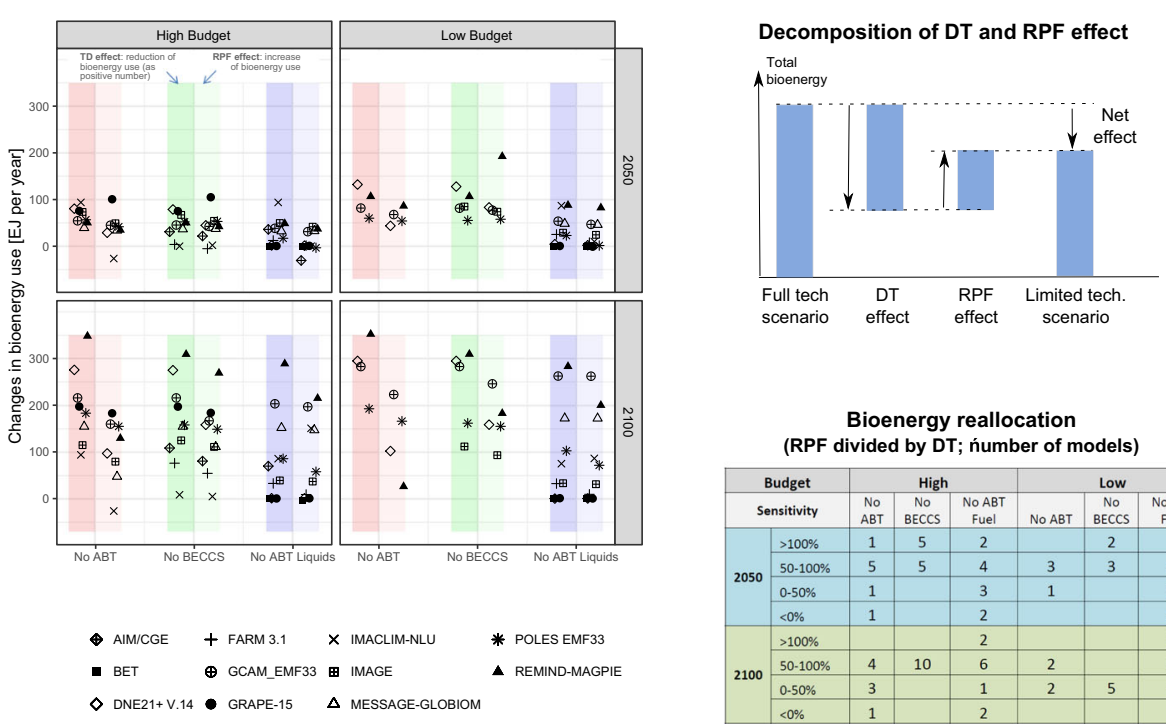

Fig. 3 Decomposition of sensitivity of ABT availability on total bioenergy use into the direct technology (DT) effect and the combined reallocation and policy feedback (RPF) effect. The small inlay illustrates the decomposition. If the DT effect is higher (lower) than the RPF effect, total bioenergy use decreases (increases) in the case with limited technology availability compared with full technology availability. Note that AIM/CGE, FARM, and MESSAGE-GLOBIOM are missing some cases due to numerical issues. The table provides summary statistics. The percentage figures in the table relate to the ratio of DT and RPF effect

option for limiting $\mathrm{CO}_{2}$ emissions. However, it only can play a limited role because total energy demand exceeds bioenergy potentials by far and needs to be complemented by other options, such as energy efficiency improvements and renewable energies.

The robustness and value of bioenergy deployment arise from the flexibility to convert biomass feedstocks into various useful energy carriers and the eventual combination with CCS, which offsets emissions from fossil fuel use. In particular, adding CCS to liquid biofuel production features a low carbon capture rate, but is preferred to electricity with high carbon capture rate because liquids are more difficult to decarbonize and demand remains substantial. Bio-liquids with CCS are a key technology that is crucial for understanding sectoral allocation of bioenergy. If it is excluded in scenarios with strong emission limitations, total bioenergy use is hardly reduced, but reallocated to other purposes like electricity generation with CCS. Large fractions of bioenergy are used with BECCS in most models, but abandoning it altogether also leads to bioenergy reallocation to technologies without CCS. If modern bioenergy use is limited to $100 \mathrm{EJ} /$ year, the use of BECCS by 2050 can increase. The reallocation of bioenergy use is reinforced by higher carbon prices that are required to comply with emission budgets under reduced mitigation technology portfolios.

Carbon prices increase over time and for tighter carbon budgets, but bioenergy use and CDR potentials approach limits that are specific to models. Adding constraints on BECCS availability leads consistently to higher carbon price increases than limiting biomass feedstock supply. Both limitations become significantly more important for tighter budgets. Only six models could project scenarios that achieve the $400 \mathrm{GtCO}_{2}$ budget, but excluding BECCS or limiting bioenergy use makes this budget infeasible for all models. Since tighter carbon budgets require earlier ramp-up of ABT deployment, the timely maturity of ABTs by 2050 is more important than the doubling of investment costs. 
A general finding is that deployment of ABTs in mitigation scenarios is a highly robust and socially valuable strategy in IAM models. However, the total use of bioenergy and its allocation is uncertain across models, but allocation is also flexible for within model sensitivity analysis. The allocative flexibility indicates that bioenergy strategies are robust, even if only few ABTs are included. The considerable sensitivity of carbon prices with respect to ABT limitations suggests that biomass feedstocks are scarce and ABTs should facilitate bioenergy uses with greatest benefit regarding both, satisfying energy demands and mitigating emissions.

From a methodological perspective, the limitations and flexibility of bioenergy use represented in IAMs are relevant when comparing it with non-IAM-based analysis. For example, the consequences of BECCS utilization are analyzed in pure technology deployment frameworks in which the link between BECCS deployment and bioenergy use is stiff (Smith et al. 2015; Anderson and Peters 2016). In such analysis, the system boundaries are narrow and do not comprise overall energy and land system effects of technology performance along with adjustments in deployment patterns, if a given emission target should be achieved and policy feedback effects need to be considered.

The results of this study on bioenergy technologies and use as a mean to achieve emission reduction targets point to a complex, multi-level policy challenge. A targeted R\&D strategy to develop ABTs, incl BECCS technologies, can help to achieve emission reduction targets with lower $\mathrm{CO}_{2}$ prices. However, the huge bioenergy demand that has to be expected by 2050 and beyond can threaten environment, food markets, and land tenureships. In default settings, IAMs assume constraints on land-use (e.g., protection of biodiversity-rich areas), but it is a real-world policy challenge to implement the corresponding regulations. Also, at the international level, climate policies must comprehensively address all emissions to avoid adverse free-riding and leakage effects (Otto et al. 2015). This is related to bioenergy trade from developing and emerging
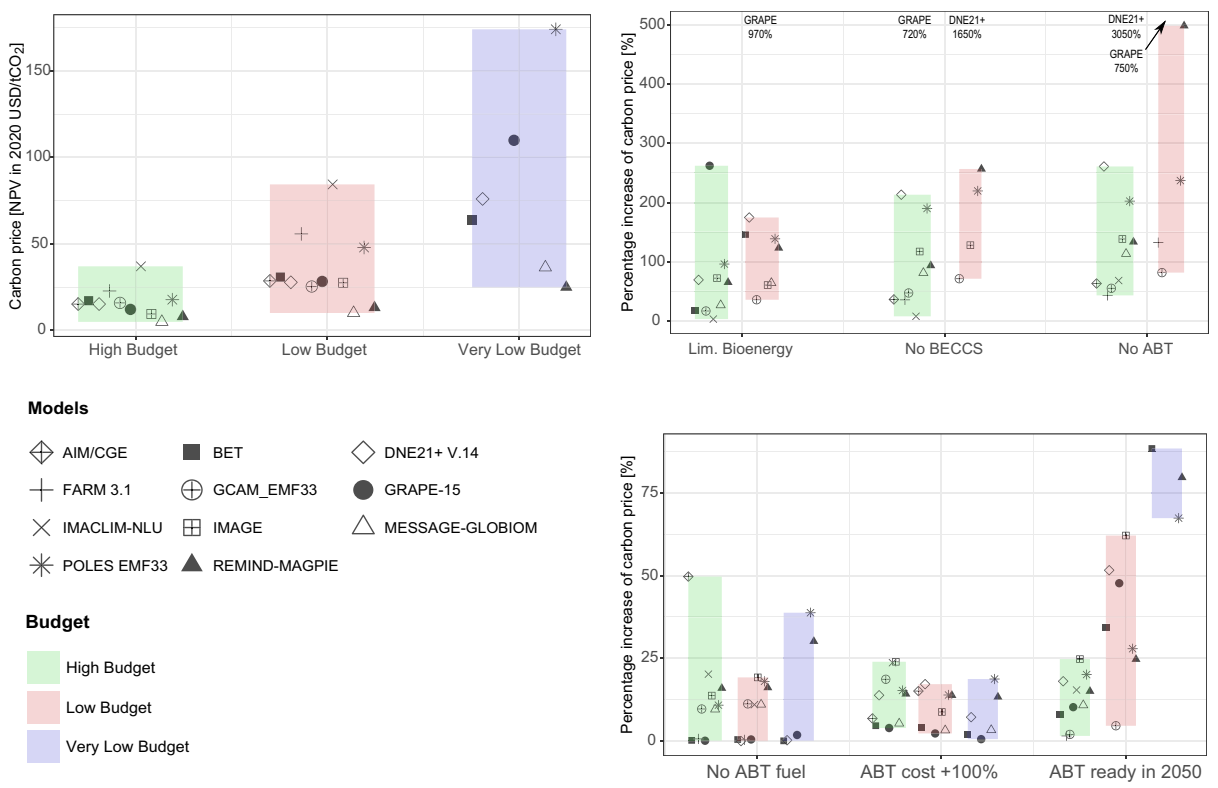

Fig. 4 PV carbon prices necessary to achieve carbon budgets. a Results for full technology cases. b, c The percentage increases for sensitivity cases. The insets in $\mathbf{b}$ indicate highly sensitive scenario variations as the numbers indicate 
economies to developed countries as is identified with the EMF-33 models. Tragically, successful R\&D policies to develop ABTs could worsen leakage effects via international bioenergy markets.

The results indicate that more research is required to assess impacts of fragmented international climate policies and failure of national land-use policies. Future comprehensive assessments would also gain from improved analysis comprising additional CDR options (Smith et al. 2015), algae biomass feedstocks (Laurens 2017), and final energy demand changes (Wilson et al. 2012) that could reduce pressure on land systems. IAMs can help to assess this complex, multi-level policy problem although not all relevant dynamics are fully represented (e.g., Buck 2016; Creutzig et al. 2013). Increased collaboration with experts from environmental, social, and political disciplines has great potential to improve IAMs in these highly relevant areas.

Acknowledgements The views expressed in this paper are those of the individual authors and do not necessarily reflect those of the author's institutions or funders. All errors are the responsibility of the authors. NB and JS received funding from the German Research Foundation (DFG) Priority Programme (SPP) 1689 (CEMICS). SR was supported by the Electric Power Research Institute (EPRI); however, the views expressed here are solely those of the authors and do not necessarily represent those of EPRI or its funders. SF and TH were supported by the Environment Research and Technology Development Fund (2-1702) of the Environmental Restoration and Conservation Agency, Japan.

\section{References}

Anderson K, Peters G (2016) The trouble with negative emissions. Science 354:182-183

Bauer N et al (2017) Shared socio-economic pathways of the energy sector-quantifying the narratives. Glob. Environ Change 42:316-330

Bhave A et al (2017) Screening and techno-economic assessment of biomass-based power generation with CCS technologies to meet 2050 CO2 targets. Appl Energy 190:481-489

Buck HJ (2016) Rapid scale-up of negative emissions technologies: social barriers and social implications. Clim Chang 139:1-13

Calvin K et al (2017) The SSP4: a world of deepening inequality. Glob. Environ Change 42:284-296

Chum H et al (2011) Bioenergy, in: IPCC Special Report on Renewable Energy Sources and Climate Change Mitigation (SRREN), Chapter 2. Cambridge University Press, Cambridge and New York

Clarke L et al (2014) Assessing transformation pathways. In: Climate Change 2014: Mitigation of Climate Change. Chapter 6. Contribution of working group III to AR5 of the IPCC. Cambridge University Press, Cambridge, United Kingdom and New York, NY, USA

Creutzig F et al (2013) Integrating place-specific livelihood and equity outcomes into global assessments of bioenergy deployment. Environ Res Lett 8:035047

Dooley JJ (2013) Estimating the supply and demand for deep geologic CO2 storage capacity over the course of the 21st century. Energy Procedia 37:5141-5150

Fargione JE et al (2010) The ecological impact of biofuels. Annu Rev Ecol Evol Syst 41:351-377

Field CB, Mach KJ (2017) Rightsizing carbon dioxide removal. Science 356:706-707

Fricko $\mathrm{O}$ et al (2017) The marker quantification of the shared socioeconomic pathway 2. Glob Environ Change 42:251-267

Fujimori S et al (2017) SSP3: AIM implementation of shared socioeconomic pathways. Glob Environ Change 42:268-283

Fuss S et al (2014) Betting on negative emissions. Nat Clim Chang 4:850-853

Grahn $\mathrm{M}$ et al (2007) Biomass for heat or as transportation fuel? Biomass Bioenergy 31:747-758

Kato E et al (2017) A sustainable pathway of bioenergy with carbon capture and storage deployment. Energy Procedia 114:6115-6123

Kaya A et al (2017) Constant elasticity of substitution functions for energy modeling in general equilibrium integrated assessment models: a critical review and recommendations. Clim Chang 145:27-40

Keramidas K et al (2017) POLES-JRC model documentation (JRC Technical Report No. EUR 28728 EN). Seville, Spain

Klein D et al (2014) The value of bioenergy in low stabilization scenarios: an assessment using REMINDMAgPIE. Clim Chang 123:705-718 
Koelbl BS et al (2014) Uncertainty in carbon capture and storage (CCS) deployment projections: a cross-model comparison exercise. Clim Chang 123:461-476

Kriegler E et al (2014) The role of technology for achieving climate policy objectives: overview of the EMF 27 study on global technology and climate policy strategies. Clim Chang 123:353-367

Kriegler E et al (2017) Fossil-fueled development (SSP5): an energy and resource intensive scenario for the 21st century. Glob. Environ. Change 42:297-315

Laurens L (2017) State of technology review—algae bioenergy (Task No. Task 39). IEA Bioenergy, Golden, CO

Lomax G et al (2015) Reframing the policy approach to greenhouse gas removal technologies. Energy Policy 78 : 125-136

Otto SAC et al (2015) Impact of fragmented emission reduction regimes on the energy market and on $\mathrm{CO} 2$ emissions related to land use. Technol. Forecast Soc. Change Part A 90:220-229

Riahi K et al (2017) The shared socioeconomic pathways and their energy, land use, and greenhouse gas emissions implications: an overview. Glob. Environ Change 42:153-168

Rogelj J et al (2015) Energy system transformations for limiting end-of-century warming to below $1.5^{\circ} \mathrm{C}$. Nat Clim Chang 5:519-527

Rose SK et al (2014) Bioenergy in energy transformation and climate management. Clim Chang 123:477-493

Sands R et al (2017) Dedicated energy crops and competition for agricultural land. Economic Research Report 223, U.S. Department of Agriculture, Economic Research Service, Washington DC

Sano F et al (2015) Assessments of GHG emission reduction scenarios of different levels and different short-term pledges through macro- and sectoral decomposition analyses. Technol. Forecast. Soc. Change Part A 90: $153-165$

Scott V et al (2015) Fossil fuels in a trillion tonne world. Nat Clim Chang 5:419-423

Smith P et al (2015) Biophysical and economic limits to negative CO2 emissions. Nat Clim Chang 6:42-50

van Vuuren DP et al (2010) Bio-energy use and low stabilization scenarios. Energy J 31:193-221

van Vuuren DP et al (2017) Energy, land-use and greenhouse gas emissions trajectories under a green growth paradigm. Glob. Environ Change 42:237-250

Waisman H et al (2012) The Imaclim-R model: infrastructures, technical inertia and the costs of low carbon futures under imperfect foresight. Clim Chang 114:101-120

Wilson $\mathrm{C}$ et al (2012) Marginalization of end-use technologies in energy innovation for climate protection. Nat Clim Chang 2:780-788

Yamamoto H et al (2014) Role of end-use technologies in long-term GHG reduction scenarios developed with the BET model. Clim Chang 123:583-596 